\title{
Hepatitis C Virus Genotyping in Saudi Population
}

\author{
Ali Hazazi ${ }^{1 *}$, Mohammed Almubarak ${ }^{1}$, Fawaz Albloui ${ }^{1}$, Omer Alsaweed ${ }^{1}$, Yousef Hawsawi $^{2,}{ }^{3}$ and Faisal \\ Alseraye $^{1 *}$ \\ 1. Department of Pathology and Laboratory Medicine, Security Forces Hospital Program, P.O. Box 3643, Riyadh 11481, Saudi \\ Arabia \\ 2. Research Center, King Faisal Specialist Hospital and Research Center, Jeddah 21499, P.O. Box 40047, Saudi Arabia \\ 3. College of Medicine, Al-Faisal University, P.O. Box 50927, Riyadh 11533, Saudi Arabia. *Authors contributed equally
}

\begin{abstract}
Background: Hepatitis C is one of the common types of viral hepatitis that threatens human life worldwide. Hepatitis C virus $(\mathrm{HCV})$ is a single-strand RNA virus that has the ability to be transmitted by contaminated blood products and intravenous drug use. $\mathrm{HCV}$ is characterized by its ability to have a high genetic diversity, which eventually results in different HCV genotypes and numerous subtypes; however, this genetic variety is a core reason for the lack of a successful vaccine for these types of viruses. Methods: HCV nucleic acid was isolated from 597 patients diagnosed with chronic hepatitis C in Security Forces Hospital (SFHR) in Riyadh. HCV nucleic acid was extracted to make the target accessible for amplification and genotype detection. The process of nucleic acid extraction and amplification was accomplished with the Abbott Molecular Systems m2000sp and m2000rt using the Abbott RealTime HCV Genotype II. Results: The findings in this study illustrated a major prevalence of genotype 4 (60\% of cases). The study also showed a large number of patients (27\%) identified as HCV genotype 1. Furthermore, the HCV genotype 1 testing detected subtypes $1 \mathrm{a}$ and $1 \mathrm{~b}$ in a number of patients. It was also demonstrated that a limited number of hepatitis $\mathrm{C}$ patients had mixed genotypes and undermined genotypes. Conclusion: The study has confirmed that HCV genotype 4 has a high prevalence when compared to the rest of the identified genotypes. Furthermore, the majority of identified genotypes and subtypes were found to be linked with age more than 50 years, which might indicate an association of HCV genotype detection with age.
\end{abstract}

Keywords: Hepatitis C, HCV virus, genotypes.

\section{Introduction}

Hepatitis $\mathrm{C}$ is a transmissible disease that is mainly caused by the hepatitis $\mathrm{C}$ virus (HCV) and seriously affects the liver [1]. HCV virus is most often transmitted via intravenous drug use and infected blood products [2]. Global statistics indicate that about 4 million cases are reported as new infections every year, and the diagnoses of these cases in a timely manner have led to the cure of up to $34 \%$ of the infected individuals [1]. Furthermore, the hepatitis $\mathrm{C}$ pathogen is known to cause both acute and chronic inflammation. Failure to diagnose or improper diagnosis of the HCV infection in its early stages is the main factor contributing to the progression of the infection to

Corresponding author: Dr. Ali Hazazi, PhD qualification. UK. Area of Research: Immunology, Molecular Pathology, Stem Cells and Cancer Studies. chronic hepatitis, which eventually may result in liver cirrhosis and hepatocellular carcinoma $[1,3]$.

$\mathrm{HCV}$ has the ability to exhibit a high genetic diversity, which outcomes in the generation of up to 7 genotypes and 67 subtypes. The genotypes are labeled with numbers 1-7, e.g., genotype 1 and genotype 2, while the subtypes are labeled with letters, such as 1a, 2b, and 1c [4]. Global HCV genotype pattern distributions vary according to the geographical regions [4, 5, 6, 7], summarized in Table1. Studying $\mathrm{HCV}$ mutations can be an important factor when determining treatment. Furthermore, the mutation that occurs in HCV is largely responsible for the complexity of creating a successful vaccine that can target and cure this type of hepatitis [8]. Several efforts have been made to reduce the morbidity of HCV so that it is not an endemic medical condition, but the genetic diversity in $\mathrm{HCV}$ remains a challenge to the elimination 
Table1 The presented data illustrate the identified HCV genotypes and the geographical regions.

\begin{tabular}{ll}
\hline HCV genotypes & Geographical regions \\
\hline HCV genotype 1 & North America, central Africa, and Europe [4, 5] \\
HCV genotype 2 & EAST Asia ,Africa [4, 5] \\
HCV genotype 3 & Asia [4, 5] \\
HCV genotype 4 & Middle East and Africa [4-6] \\
HCV genotype 5 & South Africa [4] \\
HCV genotype 6 & EAST Asia [4] \\
HCV genotype 7 & Canada [7] \\
\hline
\end{tabular}

of this risk across the world, despite the high efficacy of HCV therapy [9].

A number of epidemiological studies in Saudi Arabia with varied differences in their target population have shown an inconsistent prevalence of HCV [10]. Moreover, the studies have shown that approximately $0.9 \%$ of children and $5 \%$ of adults are reported to have $\mathrm{HCV}[11,12]$. In addition to this, the prevalence of $\mathrm{HCV}$ was found to be $50 \%$ among the infected patients on hemodialysis; however, this percentage represents only about $0.4 \%$ to $1.1 \%$ of the general population $[10,13]$. Regarding the $\mathrm{HCV}$ genotype, strain 4 and strain 1 were the most common genotypes reported in Saudi Arabia; however, these genotypes are highly pathogenic and difficult to treat [14].

The purpose of this study is to identify the distribution of HCV genotypes in patients followed at SFHR for chronic hepatitis C. The study aims to identify the association of genotype with gender and age at diagnosis. Thus, the rationale behind conducting this study is to assess the relationship between $\mathrm{HCV}$ genotypes and certain epidemiologic factors.

\section{Methods}

\subsection{Sampling}

Laboratory test results for $\mathrm{HCV}$ genotyping of a total of 597 patients followed at SFHR from January 2010 to January 2018 were reviewed. The patients' data (age, gender) were collected from the electronic laboratory records. All patients included in this study have a chronic hepatitis $\mathrm{C}$ infection.

\subsection{Procedure}

The nucleic acid extraction for HCV genotyping was performed by Abbott Molecular m2000sp. The Abbott $\mathrm{m} 2000 \mathrm{sp}$ is designed for automated sample preparation using a magnetic microparticle-based protocol (Abbott sample Preparation System). The amplification step was completed using the Abbott Molecular m2000rt RealTime System instrument. The Abbott RealTime $\mathrm{HCV}$ Genotype II is an in vitro reverse transcription-polymerase chain reaction (RT-PCR) assay for the quantification of HCV-RNA in serum. The Abbott RT-PCR HCV genotype II assay consists of two reagent kits: Abbott RT-PCR HCV Genotype II Amplification Reagent Kit and Abbott RT-PCR HCV Genotype II Control Kit. The Abbott RT-PCR HCV Genotype II assay detects genotypes 1 through 6 and subtypes $1 \mathrm{a}$ and $1 \mathrm{~b}$ with the use of genotype-specific fluorescent-labeled oligonucleotides probes (https://www.molecular.abbott/int/en/products/infectio us-disease/realtime-hcv-genotype-II). The Abbott RealTime HCV Genotyping II assays use RT-PCR to generate amplified product from the RNA genome of $\mathrm{HCV}$ in clinical specimens. In addition, an RNA sequence that is unrelated to the HCV target sequence is introduced into each specimen at the beginning of sample preparation. This unrelated RNA sequence is simultaneously amplified by RT-PCR and serves as an internal control to demonstrate that the process has progressed correctly for each sample.

\subsection{Analysis}

Quantitative variables were expressed as mean \pm 
Table 2 The presented data illustrate the identified HCV genotypes/subtypes and their association with patients' genders and ages.

\begin{tabular}{llllllll}
\hline Identified genotype & Total cases & Males & Females & M/F ratio & $\begin{array}{l}\text { Age } 20 \\
\text { years }\end{array}$ & $\begin{array}{l}\text { Age 20-50 } \\
\text { years }\end{array}$ & $\begin{array}{l}\text { Age }>50 \\
\text { years }\end{array}$ \\
\hline Genotype 4 & $356(60 \%)$ & $136(38 \%)$ & $220(62 \%)$ & $136 / 220$ & $2(0.5 \%)$ & $81(23 \%)$ & $273(77 \%)$ \\
Genotype 3 & $18(3 \%)$ & $12(67 \%)$ & $6(33 \%)$ & $12 / 6$ & 0 & $5(28 \%)$ & $13(73 \%)$ \\
Genotype 2 & $13(2 \%)$ & $2(15 \%)$ & $11(85 \%)$ & $2 / 11$ & 0 & $1(8 \%)$ & $12(92 \%)$ \\
Genotype 1 & $16(3 \%)$ & $9(56 \%)$ & $7(44 \%)$ & $9 / 7$ & 0 & $8(50 \%)$ & $8(50 \%)$ \\
Genotype 1/subtype 1a & $\begin{array}{l}82 \\
(14 \%)\end{array}$ & 39 & 43 & $39 / 43$ & 0 & 27 & 55 \\
Genotype 1/subtype 1b & $\begin{array}{l}61 \\
(10 \%)\end{array}$ & 27 & 34 & $27 / 34$ & 0 & 13 & 48 \\
Undermined genotype & $\begin{array}{l}13 \\
(2 \%)\end{array}$ & 6 & 7 & $6 / 7$ & 0 & 2 & 11 \\
Mixed Genotype 1+4 & $\begin{array}{l}36 \\
(6 \%)\end{array}$ & 20 & 16 & $20 / 16$ & 0 & 8 & 28 \\
Mixed Genotype 2+4 & $\begin{array}{l}2 \\
(0.4 \%)\end{array}$ & 1 & 1 & $1 / 1$ & 0 & 0 & 2 \\
\hline
\end{tabular}

standard deviation, and qualitative variables were expressed in terms of absolute and relative frequencies. Statistical analysis was carried out using Microsoft Excel software.

\section{Results}

\subsection{Demographic Characteristics}

The mean age \pm standard deviation of the study population was 59.4 years (min 11 years, max 92 years). The sex ratio was F/M 345/252 for this study population. The repartition of patients by age intervals showed 2 below 20 years, 145 between 20 and 50 years, and 450 more than 50 years.

\subsection{Genotype Prevalence}

The study population consisted of 356 (60\%) patients with genotype 4 . The study also contained 159 (27\%) patients with genotype 1, with subtype 1a present in $82(14 \%)$ cases, subtype $1 \mathrm{~b}$ in $61(10 \%)$ cases, and pure genotype 1 in 16 (3\%) cases. Genotypes 2 and 3 were present respectively in 13 (2\%) and $18(3 \%)$ patients. Mixed genotypes with the association of both genotypes 1 and 4, were found in 36 (6\%) patients, and the association of genotypes 2 and 4 was found in $2(0.4 \%)$ patients. The genotype was undetermined in $13(2 \%)$ cases where no specific genotype was identified (see Table 2).
3.3 Genotype Distribution per Demographic Data (Age, Gender)

\section{Discussion}

The HCV is one of the most persistent types of viral hepatitis, and several studies have found a strong link between hepatitis $\mathrm{C}$ infections and increased mortality rates [15]. In the current study of 597 patients chronically infected with $\mathrm{HCV}$ followed at SFHR revealed the predominance of HCV genotype 4 (60\%). This finding is in agreement with the few previous analyses that showed genotype 4 to be a predominant among the patients with HCV in Saudi Arabia [10, 13, 16]. Furthermore, the present study showed that the HCV genotype 1 was the next most common, while genotypes 2, 3, and 5 were the least prevalent.

A recent study in Egypt accounted a high predominance of genotype $4(94.1 \%)$ of the total HCV infections [17]. A different study in Pakistan showed the predominance of genotype $3(69.1 \%)$ followed by genotypes 1 (7.1\%), $2(4.2 \%)$, and $4(2.2 \%)$. Genotypes 5 and 6 both specified for about $0.2 \%$ [18]. On the other hand, genotype 1 was present in more than $50 \%$ of $\mathrm{HCV}$ infections in Western Europe, followed by genotype 3 in a third of the cases, with the fewest cases having genotypes 2, 5, and 6 [19]. Another study in China detected four genotypes [1-3, 6] and 17 subtypes. 
HCV genotype 1 was the most common genotype (52.45\%), followed by genotype 2 (28.70\%), genotype $3(11.66 \%)$, and genotype 6 (7.28\%) [20]. All of these reports point out that race and demographic distribution play a substantial role in the locality of HCV genotype groups.

The majority of patients in our population study were older than 50 years. This high percentage can be explained by the lack conditions of prevention of the parenteral transmission of $\mathrm{HCV}$ prior to the 1960s in Saudi Arabia [10, 21].

In this study, the results did not show a major association of any one genotype with gender; the same findings were reported earlier in Saudi Arabia [10, 11, 22]. A European study associated some patterns of HCV genotypes and subtypes with gender, especially connecting genotypes 2 and $1 \mathrm{~b}$ with female gender, while genotypes 3, 4 and 1a were more common in males [19]. Furthermore, a Chinese report linked higher subtype diversity and mixed genotypes to male gender [20]. Nevertheless, more studies might be necessary to fulfil if there is a clear link of genotype groups to the dissimilar genders.

The results also included patients who exhibited undetermined HCV genotypes in $2 \%$ of cases, where no specific genotype was detected. Recently, another study reported this finding, and this was explained by cross-reactivity or mixed infections. Sequencing the samples with undetermined genotypes might be a significant key to resolving the ambiguity regarding the undefined genotypes and identifying more subtypes [23]. Moreover, the cause of not identifying the specific genotypes could be due to other factors, including small sample quantity or more genotypes and subtypes that have not yet been revealed.

\section{Conclusion}

The study was valuable because it confirmed the few previous reports and has highlighted the $\mathrm{HCV}$ genotype distribution in Saudi Arabia. Further studies are required to elucidate the undermined $\mathrm{HCV}$ genotypes.

\section{References}

[1] Westbrook, R., and Dusheiko, G. 2014. "Natural History of Hepatitis C.” Journal of Hepatology 61 (1): S58-68.

[2] Lanini, S., Easterbrook, P., Zumla, A., and Ippolito, G. 2016. "Hepatitis C: Global Epidemiology and Strategies for Control." Clinical Microbiology and Infection 22 (10): 833-8.

[3] Navas, M., Glaser, S., Dhruv, H., Celinski, S., Alpini, G., and Meng, F. 2019. "Hepatitis C Virus Infection and Cholangiocarcinoma: An Insight into Epidemiological Evidences and Hypothetical Mechanisms of Oncogenesis." The American Journal of Pathology.

[4] Messina, J., Humphreys, I., Flaxman, A., Brown, A., Cooke, G., Pybus, O., and Barnes, E. 2014. "Global Distribution and Prevalence of Hepatitis C Virus Genotypes.” Hepatology 61 (1): 77-87.

[5] Yu, C., and Chiang, B. 2010. "A New Insight into Hepatitis C Vaccine Development." Journal of Biomedicine and Biotechnology 2010: 1-12.

[6] Pybus, O., Barnes, E., Taggart, R., Lemey, P., Markov, P., Rasachak, B., Syhavong, B., Phetsouvanah, R., Sheridan, I., Humphreys, I., Lu, L., Newton, P., and Klenerman, P. 2008. "Genetic History of Hepatitis C Virus in East Asia." Journal of Virology 83 (2): 1071-82.

[7] Murphy, D., Willems, B., Deschenes, M., Hilzenrat, N., Mousseau, R., and Sabbah, S. 2007. "Use of Sequence Analysis of the NS5B Region for Routine Genotyping of Hepatitis C Virus with Reference to C/E1 and 5' Untranslated Region Sequences." Journal of Clinical Microbiology 45 (4): 1102-12.

[8] Timm, J. 2007. "Sequence Diversity of Hepatitis C Virus: Implications for Immune Control and Therapy." World Journal of Gastroenterology 13 (36): 4808.

[9] Chidi, A., Rogal, S., Bryce, C., Fine, M., Good, C., Myaskovsky, L., Rustgi, V., Tsung, A., and Smith, K. 2015. "Cost-Effectiveness of New Antiviral Regimens for Treatment-Naïve U.S. Veterans with Hepatitis C." Hepatology 63 (2): 428-36.

[10] Bawazir, A., AlGusheri, F., Jradi, H., AlBalwi, M., and Abdel-Gader, A. 2017. "Hepatitis C Virus Genotypes in Saudi Arabia: A Future Prediction and Laboratory Profile." Virology Journal 14 (1): 208.

[11] Al Zayed, R. M., Hamdy, N. M., Al-Ajlan, H. H., and Aref, N. M. 2015. "Prevalence of HCV Genotypes and Viral Load in Saudi Arabia." International Journal of Internal Medicine 4 (2): 26-41.

[12] Shobokshi, O. A., Serebour, F. E., Skakni, L., Al-Saffy, Y. H., and Ahdal, M. N. 1999. "Hepatitis C Genotypes and Subtypes in Saudi Arabia." J. Med. Virol. 58 (1): 44-8. 
[13] Karkar, A. 2007. "Hepatitis C in Dialysis Units: The Saudi Experience." Hemodialysis International 11 (3): 354-67.

[14] Memish, Z., Knawy, B., and El-Saed, A. 2010. "Incidence Trends of Viral Hepatitis A, B, and C Seropositivity over Eight Years of Surveillance in Saudi Arabia.” International Journal of Infectious Diseases 14 (2): 115-20.

[15] Louie, K., St Laurent, S., Forssen, U., Mundy, L., and Pimenta, J. 2012. "The High Comorbidity Burden of the Hepatitis C Virus Infected Population in the United States." BMC Infectious Diseases 12 (1): 86.

[16] Abdel-Moneim, A., Bamaga, M., Shehab, G., Abu-Elsaad, A., and Farahat, F. 2012. "HCV Infection among Saudi Population: High Prevalence of Genotype 4 and Increased Viral Clearance Rate.” PLoS ONE 7 (1): 29781.

[17] Kouyoumjian, S., Chemaitelly, H., and Abu-Raddad, L. 2018. "Characterizing Hepatitis C Virus Epidemiology in Egypt: Systematic Reviews, Meta-analyses, and Meta-regressions." Scientific Reports 8 (1).

[18] Umer, M. 2016. "Hepatitis C Virus Prevalence and Genotype Distribution in Pakistan: Comprehensive Review of Recent Data." World Journal of Gastroenterology 22 (4): 1684.

[19] Kartashev, V., Döring, M., Nieto, L., Coletta, E., Kaiser, R., and Sierra, S. 2016. "HCV EuResist Study Group.
New Findings in HCV Genotype Distribution in Selected West European, Russian and Israeli Regions." J Clin Virol. 81: 82-9.

[20] Chen, Y., Yu, C., Yin, X., Guo, X., Wu, S., and Hou, J. 2017. "Hepatitis C Virus Genotypes and Subtypes Circulating in Mainland China." Emerging Microbes \& Infections 6 (1): 1-7.

[21] Khan, A., Al Balwi, M., AlAyyar, L., AlAbdulkareem, I., Albekairy, A., and Aljumah, A. 2017. "Tracing the Epidemic History of Hepatitis C Virus Genotypes in Saudi Arabia." Infection, Genetics and Evolution 52: 82-8.

[22] Al-Faleh, F., Huraib, S., Sbeih, F., Al-Karawi, M., Al-Rashed, R., Al-Mofleh, I., Sougiyyah, M., Shaheen, M., and Ramia, S. 1995. "Hepatitis C Virus Genotypes in Patients with Chronic Liver Disease and Haemodialysis Patients from Saudi Arabia." Journal of Viral Hepatitis 2 (6): 293-6.

[23] Mesbahi, Z., Kabbaj, H., Malki, H., Bouihat, N., Qrafli, M., Belefquih, B., Marcil, S., Challine, D., Pawlotsky, J., Bouvier, M., and Seffar, M. 2018. "Indeterminate Genotypes of Hepatitis C Virus by the Abbott RealTime HCV Genotype II Assay in Morocco. About Eight Cases Resolved by a Sequencing Method." Journal of Medical Virology 90 (8): 1352-7. 